\title{
Analysis of Different Pheromone Decay Techniques for ACO based Routing in Ad Hoc Wireless Networks
}

\author{
Sharvani G S \\ R.V College of Engineering \\ Bangalore
}

\author{
A G Ananth, PhD. \\ R.V College of Engineering \\ Bangalore
}

\author{
T M Rangaswamy, PhD. \\ R.V College of Engineering \\ Bangalore
}

\begin{abstract}
Ant Colony Optimization (ACO) technique deals with exploratory behavior of ants while finding food by following a path based on the concentration of the pheromone. A major limitation with ACO algorithm is "stagnation". This occurs when all ants try to follow same path to reach the destination due to higher pheromone concentration and causes congestion when applied to Adhoc Wireless Network (AWN). In the present paper, a detailed analysis of ACO based different pheromone decay techniques such as Discrete, Exponential and Polynomial has been carried out. Pheromone intensity and probability of choosing path for packet transmission are used as parameters for the analysis. It is found that the Discrete decay is not preferable for Congestive network as it leaves large amount of pheromone traces. The polynomial decay technique choose better path and avoid longest path which lead to delay at the time of packet delivery. The Exponential decay has been found to exhibit better performance compared to Discrete and Polynomial decay techniques, However it loses the pheromone traces very fast. The Efficient fine tuning of the exponential decay model can be achieved by using stability factor ' $\Delta$ '. The present analysis shows that for values of ' $\Delta$ ' $<0.08$ the probability of selection of the longest optimal paths is $<1 \%$, where as for ' $\Delta$ ' $>0.09$ the probability of selection of the longest optimal path increases to $18 \%$.. The introduction of the stability factor ' $\Delta$ ' improves AWN performance in terms of packet delivery. The results are presented and discussed in the present paper.
\end{abstract}

Keywords: Ad Hoc wireless Networks, Swarm Intelligence, Ant Colony Optimization, Stagnation, Pheromone decay

\section{INTRODUCTION}

Ad Hoc Wireless Networks (AWN) is an autonomous system with no centralized entity controlling the nodes. At the time of communication, nodes rely on many intermediate nodes. Hence the nodes should be more intelligent in the form of a host as well as a router (to forward the nodes). Routing in MANET is a challenging task due to characteristics like node mobility - which leads to dynamic Topology, Error prone shared channel, channel contention and limited device availability. Hence, an efficient routing protocol capable of reducing control overheads and efficient bandwidth utilization needs to be developed. Ant Colony Optimization (ACO) is a Swarm Intelligence (SI) based routing algorithm proposed for high quality routing in AWN.

\section{Ant Colony Optimization (ACO)}

ACO was inspired by an algorithm called "Ant System (AS). An ant system chooses the best path laid by the previous ant, which went in search of food and has returned back. ACO deals with artificial systems, which are inspired from food foraging behavior of real ants, which can find optimal solutions inspite of changes in the environment. The main idea is indirect communication between the ants by means of pheromone trails, which helps them to find shortest path between their nest and food. ACO are currently applied to applications, such as graph coloring problems, scheduling problems, traveling sales man problem, network routing problem, clustering, robotics etc[1][2][3].

A major problem with ACO algorithm is "stagnation". This occurs when all ants try to follow same path to reach the destination (since there is more pheromone). This when applied in AWN comes to a convergence state (equilibrium) and attracts all the data packets to follow the same path, which leads to congestion. The next packet without aware of the congestion follows a non-optimal path and loses its packets due to frequent packet drops [4] [5] [6] [7].

\section{Techniques to alleviate Stagnation in ACO}

To mitigate the above problem of ACO algorithms, different methodologies where adopted and are categorized as follow [8].

\section{- $\quad$ Privileged pheromone Laying \\ - Pheromone-heuristic control \\ - Pheromone Control}

In privileged pheromone laying approach-selected subset of ants are used to update pheromone values on the best path. This reduces the probability of ants following the stagnant paths that are non-optimal and congested due to overload. In the next approach Pheromone-heuristic control, ants not only try find best path based on pheromone concentration on that edge, but also the other factors like queue length, delay and distance. These factors alter the selection of the best path and avoid the stagnation.

Last approach for stagnation problem is controlling the pheromone concentration. Pheromone control can be done in many ways. They are as follows

\section{- Evaporation \\ - $\quad$ Aging \\ - Limiting Pheromone \\ - Smoothing Pheromone}

These approaches discourage non-optimal paths and reduce the influence from past experience. In evaporation approach all the edges with certain pheromone concentration evaporates as the time increases. This is done by an evaporation factor call ' $p$ '. Evaporation not only removes the stale entries, it also balances pheromone concentration in optimal paths. This helps other ants to survey new paths (better).Aging is another technique to reduce stale entries in the network. In aging 
'older ants are deposits lesser pheromone as compared to younger ants. Older ants are those ants that have taken longer time to reach the destination. Both the techniques aging and evaporation aims at finding new best path when there is congestion. Pheromone can be limited for every edge by placing an upper bound [9] [10]. A variant of the Limiting pheromone is pheromone smoothing in this approach the pheromone is increased along an edge as follows.

$\tau \mathrm{i}, \mathrm{j}\left(\mathrm{t}^{\prime}\right)=\tau \mathrm{i}, \mathrm{j}(\mathrm{t})+\delta^{*}(\tau \max -\tau \mathrm{i}, \mathrm{j}(\mathrm{t}))$

Where $\delta$ varies from 0 to 1

It is also observed that smaller amount of pheromone is deposited gradually until the upper bound is reached. Evaporation is done in a uniform manner on all edges. This technique seems to be more effective in avoiding the generation of dominant path.

\section{Stagnation avoidance algorithms}

Elitist Ant System (EAS) algorithm is a variation of ACO [11]. Here pheromone deposits are only for the best path found. The major problem with this algorithm is early stagnation. Max-Min Ant systems (MMAS) [12] overcome the early stagnation problem by adding an extra constraint which says that the pheromone is bounded between maximum and minimum pheromone concentration.

Stagnation avoidance algorithm was developed while ACO found its application in Scheduling of flexible manufacturing systems [13]. The methodology developed deals with quick convergence and stagnation avoidance in which parameters of ACO such as evaporation co-efficient which control the trail and its visibility are fine tuned. MMAS was further improved by adopting Minimum Pheromone Threshold Strategy (MPTS) [14]. Here the bound between maximum and minimum threshold is fine tuned based on performance of the network. Improved Lower Limits for Pheromone Trails in ACO adopts improved estimates of the lower pheromone value. This helps algorithms like MMAS (sets implicit pheromone trail limit) to avoid stagnation [15]. Another work on stagnation avoidance for scheduling of real-time tasks [16] uses a non-preemptive scheduling approach based on distance function as an extra parameter in the transition rule with the pheromone information.

A framework for stagnation avoidance was developed while adapting ACO in TSP [17]. This frame work evaluates each solution against the last "N" solutions globally constructed by ants. As soon as "N" solutions are available, their moving average " $A$ " is computed. New value of A is compared with old value. If New A is lower than old, the trail level of the last solution's moves is increased, otherwise it is decreased.

An ACO algorithm with improved pheromone correction strategy for the Minimum Weight Cover Problem (MWCP) [18] [19] explains that new Hybridization techniques. Corrections to the pheromone trails are performed based on suspicion whether it is good or bad. It decrements the pheromone trails very fast if it finds more suspicious elements. In Hybrid architecture, the age of the ant decides whether the trail should be increased or not [20]. Another solution for stagnation was found by using information entropy [21] which helped the algorithm to fine tune its pheromone trails for local search.

\section{Design of the Algorithm}

The network is represented as $\mathrm{G}=(\mathrm{V}, \mathrm{E})$, a connected graph with ' $\mathrm{V}$ ' nodes and an edge ' $\mathrm{E}$ ' connecting two nodes. An edge is directional, starting at a vertex $\mathrm{x} \in \mathrm{V}$ and ending at $\mathrm{y}$ $\in \mathrm{V}$. Thus, each edge is described by an ordered pair of vertices $e=\{x, y\} \in E$. The neighbors of node ' $x$ ' can be defined as a set $S^{x}$ containing all other nodes for which ' $\mathrm{x}$ ' are the starting point and other nodes as ending points $; S^{x}=\{y \in$ $\mathrm{V}:\{\mathrm{x}, \mathrm{y}\} \in \mathrm{E} ; \mathrm{x} \notin \mathrm{S}^{\mathrm{x} ;} \mathrm{S}^{\mathrm{x}} \subset \mathrm{V}$.Every pair of links between two nodes can be thought as bidirectional link if $\mathrm{x} \in \mathrm{S}^{\mathrm{y}}$ and $\mathrm{y}$ $\in \mathrm{S}^{\mathrm{x}}$. The metric of optimization is number of hops between the nodes. In order to apply trail update/decay in networks routing, routing tables are replaced by tables of probability, which are also called as pheromone tables, as shown in Table 1. These probabilities are used by ants to explore network and possibly find new and better routes.

The goal of MANET is to find the shortest path between source node $V_{s}$ and destination $V_{d}$, where $V_{s}$ and $V_{d}$ belong to $\mathrm{V}$. The path length is given by the number of nodes along the path. Each node maintains a table keeping track of the amount of pheromone on each neighbor edge/ link e $(i, j)$ represented as $\varphi_{i, j}$.

The table may be visualized as a matrix with neighbor nodes $\mathrm{N}_{\mathrm{i}}$ forming the labels for the rows and destination nodes forming the labels for the labels for the columns. Table Size, Pheromone Updation, Pheromone Decay are the various factors taken care in maintaining the pheromone table.

\begin{tabular}{|l|l|l|l|l|l|}
\hline \multirow{2}{*}{$\begin{array}{l}\text { Neighbor } \\
\text { Node }\end{array}$} & \multicolumn{4}{|l|}{ Destination Nodes } \\
\cline { 2 - 6 } & 1 & 2 & 3 & $\ldots \ldots \ldots \ldots$ & N \\
\hline 1 & P11 & P12 & P13 & $\ldots \ldots \ldots \ldots$ & P1N \\
\hline 2 & P21 & P22 & P23 & $\ldots \ldots \ldots \ldots$ & P2N \\
\hline 3 & P31 & P32 & P33 & $\ldots \ldots \ldots \ldots$ & P3N \\
\hline$\ldots \ldots \ldots \ldots .$. & $\ldots \ldots$ & $\ldots \ldots$ & & $\ldots \ldots \ldots \ldots$ & \\
& & & & & \\
\hline L & PL1 & PL2 & PL3 & $\ldots \ldots \ldots \ldots .$. & PLN \\
\hline
\end{tabular}

Table 1 Pheromone table for node ' $V_{i}$ '

Table of probabilities and the array can be seen as memories local to nodes capturing different aspects of the network dynamics. The latter maintains absolute time estimates to all the nodes, while the former gives relative probabilistic goodness measures for each link-destination pair under the current routing policy implemented over the entire network. follows

The probability at node $\mathrm{V}_{\mathrm{i}}$ can be computed as

$=0 \quad\left\{\begin{array}{l}\varphi_{i, j} /\left(\Sigma \varphi_{i, j}\right) \quad \text { for } \mathrm{j} \varepsilon N_{i} \\ \text { for does not belong to } N_{i}\end{array}\right.$

The probability $\mathrm{p}_{\mathrm{i}, \mathrm{j}}$ of a node $\mathrm{V}_{\mathrm{i}}$ has the constraint that $\Sigma \mathrm{p}_{\mathrm{i}, \mathrm{j}}=1$ $\mathrm{j} \varepsilon \mathrm{N}_{\mathrm{i}}$. The value of $\varphi_{\mathrm{i}, \mathrm{j}}$ is incremented by ' $\gamma$ ' by the ant packet which move along the path $V_{i}$ to $V_{j}$. That is $\varphi_{i, j}=\varphi_{i, j}+\gamma$. The 
concentration $\varphi_{\mathrm{i}, \mathrm{j}}$ indicates the usage of the link. Parallel to it the concentration of the pheromone should decrease with time, at every constant interval ' $t$ ', the value of $\varphi_{i, j}$ between the nodes $V_{i}$ to $V_{j}$ is decreased by Decay Factor(DF). The rate of increase in pheromone $(\gamma)$ greater that the rate of decrease in pheromone. [22].

Pheromone Decay: The pheromone intensity of each path in the network is decayed or evaporated continuously using different decay techniques. Pheromone evaporation is necessary in order to avoid local maxima problem. Three different decay techniques used in the project are Discrete decay, Polynomial Decay and Exponential Decay. Different techniques are selected (which decays approximately same when the node mobility is static) based on the choice made by the user as shown in Eqn(1), Eqn (2) and Eqn(3).

\section{Technique 1: Discrete decay}

$$
\varphi_{\mathrm{i}, \mathrm{j}}=\varphi_{\mathrm{i}, \mathrm{j}}\left(\varphi_{\mathrm{i}, \mathrm{j}}-0.1,0\right) ; \ldots \ldots \ldots \ldots \ldots \ldots \ldots \ldots \ldots \ldots \ldots \ldots \text { Eqn }(1)
$$

\section{Technique 2: Polynomial decay}

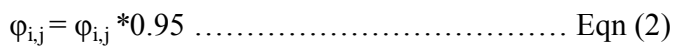

\section{Technique 3: Exponential decay}

$$
\varphi_{\mathrm{i}, \mathrm{j}}=\varphi_{\mathrm{i}, \mathrm{j}} * \mathrm{e}^{-\tau} ;
$$

Different parameters used for analysis are pheromone intensity and probability of choosing path. With this methodology, it is found that the decay techniques become application specific [23].

\section{Efficient fine tuning pheromone technique to alleviate stagnation problem}

Evaporation is fine tuned based on the stability factor of the node in the vicinity of the node that forwards the packet. During packet forwarding time, each node calculates the 'hello' sent and 'hello' received by its neighboring nodes and calculates the stability ratio shown in Eqn (4)

$$
\Delta=(\text { Hello Replied / Hello Sent }) * 100 \text {.........Eqn (4) }
$$

With the help of ratio calculated, evaporation is controlled for each neighboring node by fine-tuning the decay speed like decaying fast for unstable nodes and decaying little slower for stable nodes as shown in Eqn (5). Exponential Decay may result in faster lost of pheromone trail traces. Pheromone trail is controlled by adding an extra parameter ' $\Delta$ ' to the exponential decay factor.

$$
\varphi_{\mathrm{i}, \mathrm{j}}=\varphi_{\mathrm{i}, \mathrm{j}} \cdot\left(\mathrm{e}^{-\tau+\Delta}\right) \ldots \ldots \ldots \ldots \ldots \ldots \ldots \ldots \ldots \ldots \ldots \text { Eqn }(5)
$$

This technique helps the AWN to overcome stagnation problem, it also reduces the control overhead and have efficient packet delivery ratio. ' $\Delta$ ' is tunable based on the application and the node mobility.

\section{RESULTS AND DISCUSSIONS}

The pre-processing is done using MATLAB. Prebuilt functions are used wherever possible. Customized programs are written in $\mathrm{C} / \mathrm{C}++$ to understand and fine tune the mathematical operations. However, using pre-built functions, as opposed to writing, debugging, and validating our own functions, will save a great deal of programming time. Three different decay techniques Polynomial, Discrete and Exponential were used to measure change of probability and pheromone intensities for the selecting an optimal path

A Major problem in ACO is stagnation which causes heavy traffic on the optimal path. To avoid this, saturation level for pheromone ( 0.89 found to be more appropriate as compared to other values tested) is kept, to avoid congestion. If the pheromone level has reached the saturation limit then the next optimal path is chosen to forward the packets. The parameters, change of probability and pheromone intensities of the selecting a new path are analyzed with and without saturation technique.

\section{Discrete Decay technique:}

Using the discrete decay technique, the Probability of optimal path selection and the pheromone intensity with saturation has been determined and plotted against the simulation time and shown in figures 9 (a) and 9 (b). The shortest path (Blue), the Intermediate path (Green) and the longest path (Red) calculated in terms of delay is also shown in the same figure. The figure 9(a) shows the pheromone intensity profiles as derived for discrete decay pheromone intensity (Eq.1) as a function of simulation time for the three different optimal paths. The figure 9 (b) shows the probability of selection of different optimal paths namely short, intermediate and long paths for data packet flow. It can be seen from the figure 9(a) that in the shortest path, the pheromone intensity first increases rapidly and then saturates with the intensity becoming constant with time. Where as the figure 9(b) shows that the probability of short optimal path selection first reaches a maximum value of 0.89 and start decaying with time by a factor of $(0.35)$ when the pheromone intensity remains constant.. When the shortest path is found, most of the data packets follow that path.

It is also seen from the figure that the probability of the intermediate optimal path increases $(0.2)$ during the saturation phase of the pheromone intensity and the data packets also choose intermediate path for propagation. It is also found that during the saturation phase of pheromone intensity the probability of selection of longest optimal path increases by a small amount $(0.15)$ and some data packets also choose the longest optimal path for propagation. The discrete decay of pheromone intensity shows that for both intermediate and the longer optimum paths probability is significant and both the paths are chosen for data packets propagation in the network.

\section{Polynomial Decay technique}

Analysis is performed using the Polynomial decay technique as shown in Figure 10 (a) and 10 (b). It can be seen from the figure 10 (a) that in the shortest path, the pheromone intensity first increases rapidly and then saturates with the intensity becoming constant with time. Whereas the figure 10 (b) shows that the probability of short optimal path selection (first reaches a maximum value 0.85 and start decaying with time by a factor of 0.25 when the pheromone intensity remains constant. When the shortest path is found, most of the data packets follow that path. It is also seen from the figure 
that at the same time, the probability of the intermediate optimal path increases by a factor of 0.2 during the saturation phase of the pheromone intensity and the data packets also choose intermediate path for propagation.

It is also found that during the saturation phase of pheromone intensity the probability of selection of longest optimal path increases by a very small amount 0.02 and some data packets also choose the longest optimal path for propagation. The Polynomial decay of pheromone intensity shows that for both intermediate and the longer optimum paths probability is significant and both the paths are chosen for data packets propagation in the network.

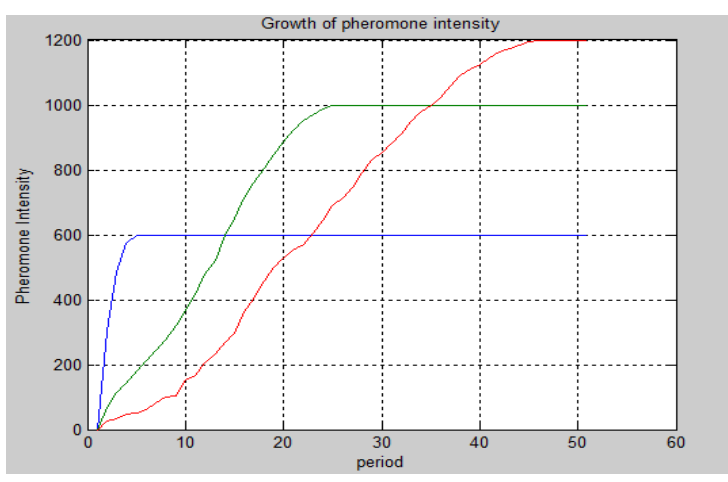

Fig 9(a) pheromone intensity vs period

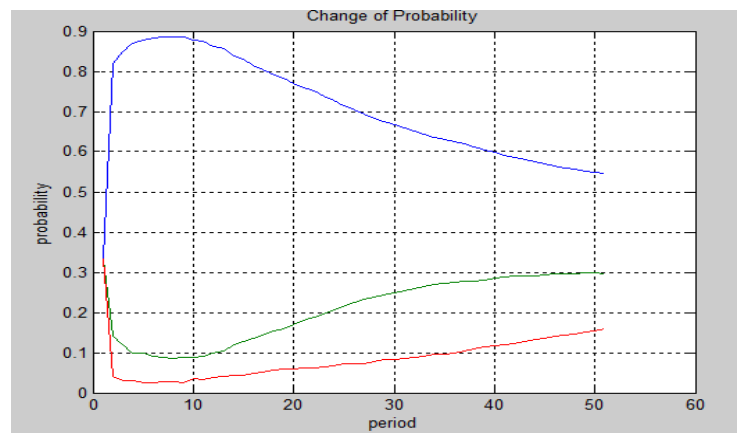

Fig 9 (b) probability of path selection vs period

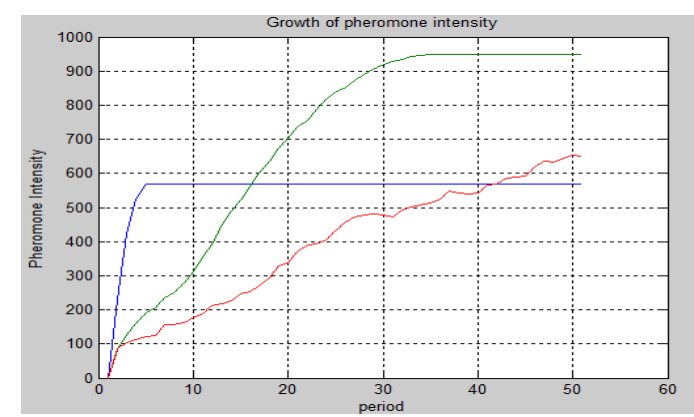

Fig10(a):Pheromone intensity vs. period

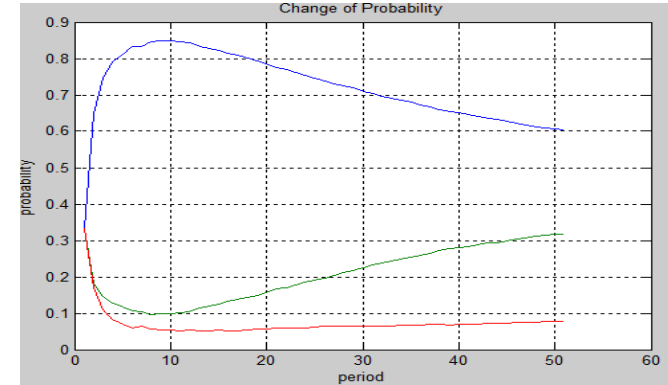

Fig 10(b) probability of path selection vs. period

\section{Exponential Decay technique:}

Similar analysis is performed Using the Exponential decay and shown in figures 11 (a) and 11(b).

It can be seen from the figure 11 (a) that in the shortest path, the pheromone intensity first increases rapidly and then saturates with the intensity becoming constant with time. Whereas the figure 11 (b) shows that the probability of short optimal path selection first reaches a maximum value 0.85 and start decaying with time by a factor of 0.15 when the pheromone intensity remains constant.. When the shortest path is found, most of the data packets follow that path. It is also seen from the figure that at the same time, the probability of the intermediate optimal path increases by a factor of 0.1 during the saturation phase of the pheromone intensity and the data packets also choose intermediate path for propagation.

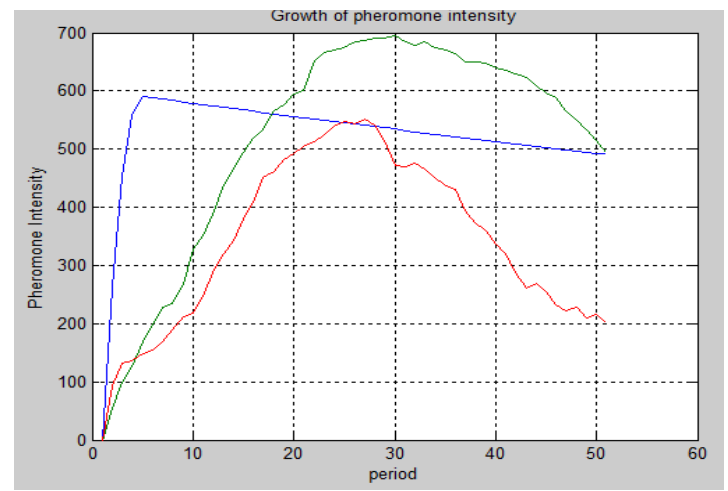

Fig 11 (a) pheromone intensity vs period

It is also found that during the saturation phase of pheromone intensity the selection probability of the longest optimal path does not change and remains constant, which indicates that there is no data packet flow in the longest optimal path. The Exponential decay of pheromone intensity shows that intermediate path probability is significant and only intermediate path is chosen for data packets propagation in the network.

The probability of selection of optimum paths for the three decay techniques is summarized in Fig 12. It may be noted from the Figures 9, 10 and 11 and 12 that the probability of selection of the longest optimal path for discrete pheromone decay is $\sim 0.15$, for polynomial decay reduces to $\sim 0.02$ and becomes almost zero for exponential decay of pheromone intensity. This leads to the elimination of data packet flow in the longest optimal path for the exponential pheromone decay. In this case it is found that most of the data packet flow follows the shortest optimal path and intermediate path, thus prohibiting the longest path for the data packet 
flow. From these findings it may be suggested that the exponential decay model for the pheromone intensity is more efficient compared to the discreet or polynomial decay models for AWN networks.

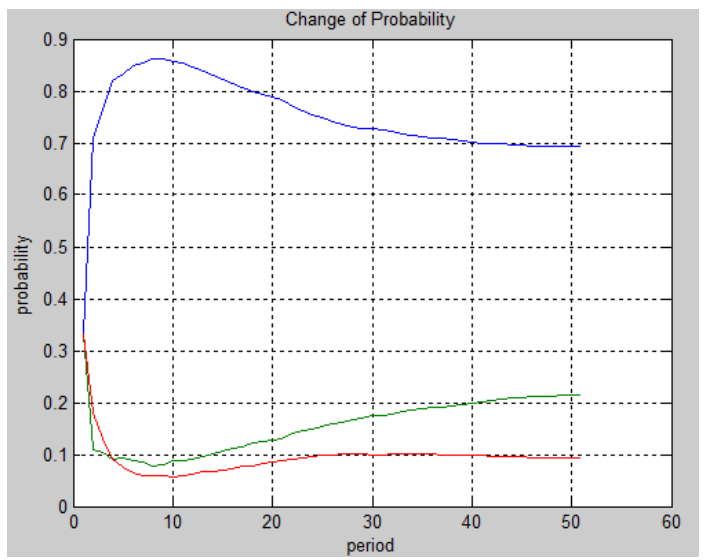

Fig 11(b) probability of path selection vs period

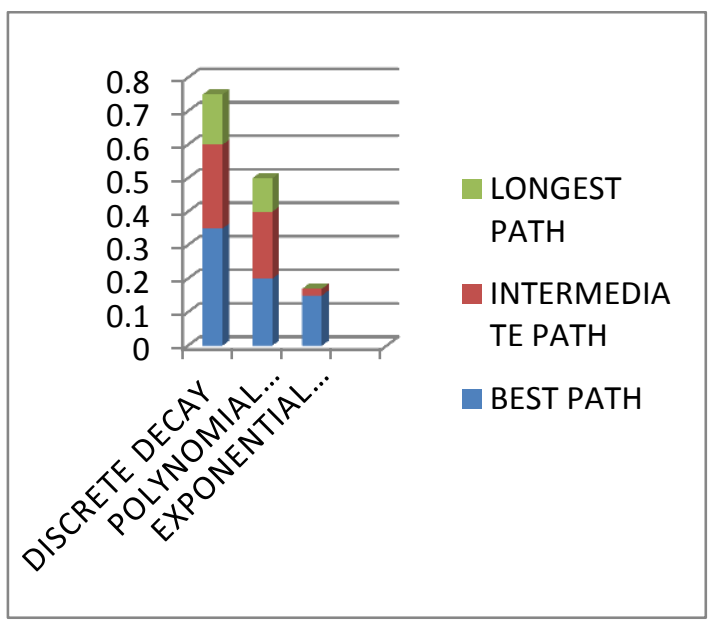

Fig 12 Analysis of Different decay techniques

Out of the many decay functions analyzed the exponential decay techniques in ACO is more applicable for dynamic networks like AWNs. The limitation of this technique is pheromone evaporates (uniformly) very quickly after some time leaving no traces for the data transmission. Hence, fresh route discovery is established to know the destination address. This causes more control overhead and makes bandwidth inefficient. To deal with this problem, controlled exponential evaporation is adopted which fine tunes evaporation based on the stability of the node.

\section{Efficient fine tuning pheromone technique}

The analysis has been continued further by using different values of stability factor ' $\Delta$ ' to establish the probability of selecting the best path in the network for the exponential decay of pheromone by fine tuning of pheromone concentration to alleviate stagnation problem. The stability ratio ' $\Delta$ ' has been varied from 0.001 to 0.1 to determine the optimum value of ' $\Delta$ ' that can be used for confining the data pocket flow to the shortest (Blue) and the intermediate optimum paths.
Fig 13 shows the variation of the probability of selecting the best path for different values of ' $\Delta$ '. It is clearly evident from the table that for the lower range of values of ' $\Delta$ ' between $0.001-0.008$ the probability of selecting the longest optimum path (Red) remains very low $<1 \%$ comparable to exponential pheromone decay. This indicates that for ' $\Delta$ ' values $<0.008$ the flow of data pockets are confined to the shortest and the intermediate optimum paths and there is no data packet flow in the longest optimum path.

For values of ' $\Delta$ '> 0.09 the probability of selection of optimal path for shortest path and the intermediate path decreases dramatically and it is found that the packet flow on the longest path starts increasing rapidly. This indicates that the probability of selection of optimal path for the exponential decay is limited by the values of ' $\Delta$ '.

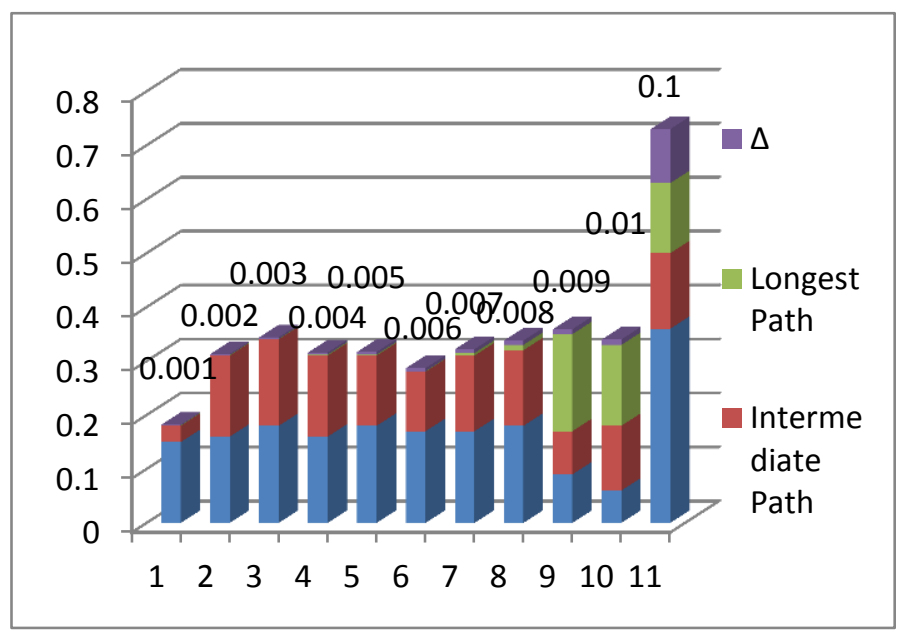

Fig 13: Analysis of Different stability factor ' $\Delta$ '

The flow in different paths can be controlled by changing the values of ' $\Delta$ ' between till $0.001-0.008$ for which the packet flow remains confined to shortest and the intermediate paths. Once the ' $\Delta$ ' value increases above 0.009 the data packet flow in the longest path increases rapidly and the packet flow in three different paths becomes closer to discrete Pheromone concentration decay model.

Further to highlight the observation made in the analysis, the pheromone intensity and the probability of selection of the three optimal paths namely shortest (Blue), Intermediate (Green) and longest (Red) paths are plotted for the two different values of ' $\Delta$ ' $=0.08$ and 0.09 and shown in figures 14(a),(b) and Fig 15(a),(b).

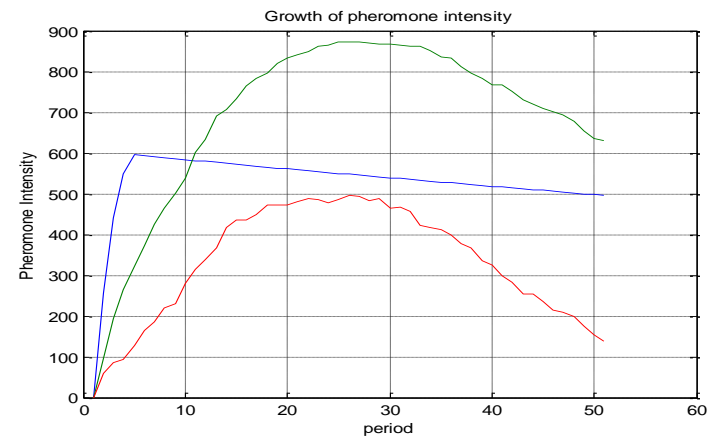

Fig 14(a): pheromone intensity vs period 


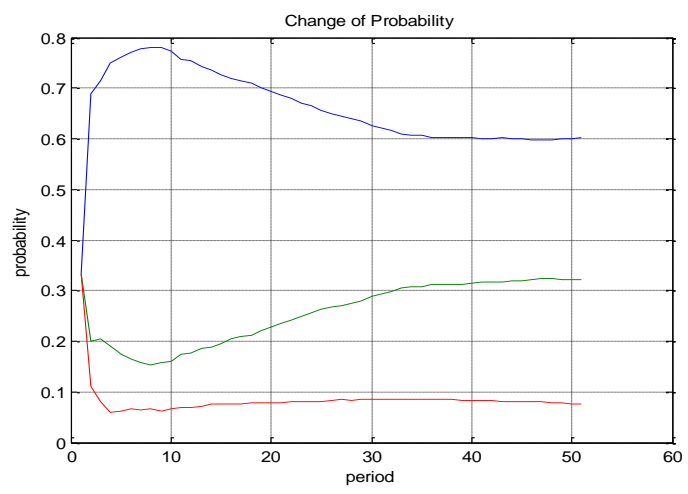

Fig 14(b): probability of path selection vs period

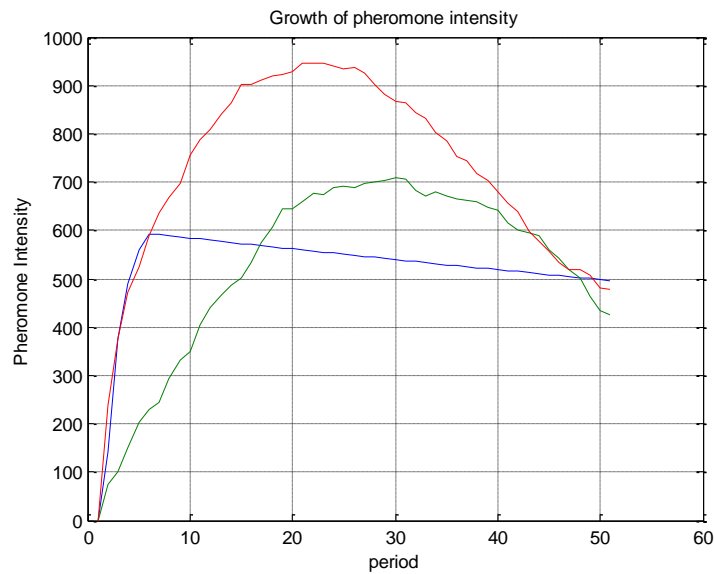

Fig 15(a): pheromone intensity vs period

It is clearly seen in the figure 14 (b) for values of ' $\Delta$ ' $=0.08$ the probability of selection of the longest optimal paths is $<1 \%$, where as for the ' $\Delta$ ' $=0.09$ as seen in the figure $15(\mathrm{~b})$ the probability of selection of the longest optimal path increases to $18 \%$. This indicates that for ' $\Delta$ ' $>0.09$, the exponent decay model for the pheromone concentration is not adequate to control the data packet flow in the AWN networks.

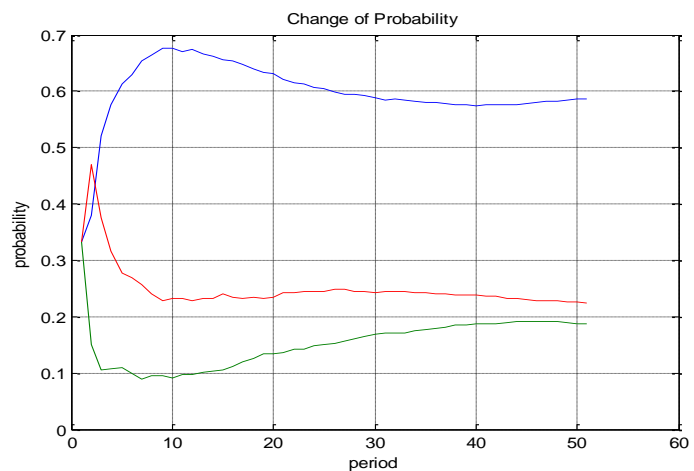

Fig 15(b): probability of path selection vs period

\section{CONCLUSIONS}

From the results and discussions presented, the following conclusions can be drawn
1. The Discrete, Polynomial and Exponential Pheromone Decay model applied to MANET indicates that the Exponential Decay model gives a better performance compared to the other two models. With Exponential Pheromone Decay model the probability of data packet flow is mostly confined to shortest and intermediate optimum paths and the data packet flow in the longest path is almost negligible.

2. The exponential pheromone decay process can be controlled by fine tuning the decay rates by introducing the node stability factor ' $\Delta$ '.

3. For values of ' $\Delta$ ' $=0.001$ to 0.008 , the data packet flow is mostly confined to the shortest and the intermediate optimal paths. Beyond values of ' $\Delta$ '> 0.008 , the data packet flow data significantly increases in the longest path. For higher values of ' $\Delta$ ', the Exponential Pheromone Decay model will start behaving like Discrete Pheromone Decay model i.e. the data packets will follow even the longest path.

\section{REFERENCES}

[1]. Bonabeau, E, Dorigo M and Theraulaz G, "Swarm Intelligence from Natural toArtificial Systems", Oxford University Press, 1999.

[2]. Bonabeau, E, Dorigo M and Theraulaz G, “ Inspiration for optimization from social insect behavior, Vol 406 , 39-42, 0028-0836, 2000

[3]. Di Caro G, Ducatelle F and Gambarella L M, "AntHocNet" Ant-based HybridRouting algorithm for MANETs", In : IDSIA-25-04-2004 Technical Report , 112 Dalle Molle Institute for Artificial Intelligence, Switzerland , 2004

[4]. F Neuman D Sudholt, C Witt," Rigorous analyses for the combination of ant colony optimization and local search", ANTS 2008, Proceedings of the 6th International Conference on ACO and Swarm Intelligence, Springer-Verlag, Berlin, pp 132-143, 2008

[5]. Rajagopalan S and Shen C C, “ANSI:"A swrm Intelligence-based unicast routing protocol for Hybrid AWN,", Journal of System Architecture, Special issues on Nature Inspired Applied Systems, 2007, 485-504

[6]. Saleem M and Farooq M. A frame work for empirical evaluation of nature inspired routing protocols for wireless sensor networks", In proceedings of the IEEE congress on evolutionary computing, PP 5751-758, 2007.

[7]. Saleem M, Khayam S and Farooq M," A formal performance modeling framework for bio Inspired ad Hoc routing protocols", in ACM GECO, PP 103-110, New York Acm, 2008.

[8]. Dorigo M, Birattari M and Stuzle T," Ant Colony Optimisation.Artificial Ants as a computational Intelligence Technique “, Technical Report, IRDIA, 112, 1781-3794, 2006

[9]. De Rango F , Tropea M , Provato A, Sanmaria A F and Marano S , "Minimum Hop Count and Load Balancing Metrics based on Ant Behaviour over HAP Mesh", IEEE GLOBECOMM, pp 1-6, New Orleans, 2008. 
[10].De Rango F , Tropea M," Energy saving and Load balancing in wireless adhoc networks through ant basaed routing”, SPECTS, Vol 41, 978-1-2-4244-4165-5,2009

[11].Ducatelle F, Di Caro G and Gambardella L M, "Principles and applications of Swarm Intelligence for adaptive routing in telecommunications networks", Swarm Intelligence, 2010.

[12].T Stutzle, H H Hoos ,'Max-Min Ant system" Future Generation Computing Syst,(2000), PP 889-914.

[13].R Kumar, M K Tiwari and R Shankar, "Scheduling of flexible manufacturing systems: an ant colony optimization approach", Proceedings Instn Mech Engrs Vol 217, Part B: J Engineering Manufacture, 2003,pp 1443-1453.

[14].Kuan Yew Wong, Phen Chiak See, " A New minimum pheromone threshold strategy(MPTS) for Max-min ant system “, Applied Soft computing, Vol 9, 2009, pp 882888

[15].David C Mathew, "Improved Lower Limits for Pheromone Trails in ACO", G Rudolf et al (Eds), LNCS 5199, pp 508-517, Springer Verlag, 2008.

[16].Laalaoui Y, Drias H, Bouridah A and Ahmed R B, “ Ant Colony system with stagnation avoidance for the scheduling of real time tasks", Computational Intelligence in scheduling, IEEE symposium, 2009, pp 16.

[17].E Priya Darshini, " Implementation of ACO algorithm for EDGE detection and Sorting Salesman problem", International Journal of Engineering science and Technology, Vol 2, pp 2304-2315, 2010
[18].Alaa Alijanaby, KU Ruhana Kumahamud, Norita Md Norwawi, "Interacted Multiple Ant Colonies optimization Frame work: an experimental study of the evaluation and the exploration techniques to control the search stagnation", International Journal of Advancements in computing Technology Vol 2, No 1, March 2010, pp 78-85

[19].Raka Jovanovic and Milan Tuba, “ An ant colony optimization algorithm with improved pheromone correction strategy for the minimum weight vertex cover problem", Elsevier, Applied Soft Computing, PP 53605366,2011 .

[20].Priyanka Sharma, Dr K Kotecha, “ Optimization in stagnation avoidance of ACO based routing of Multimedia Traffic over Hybrid MANETs", International Journal of computer science and technology, IJCST, Issue 2, ISSN: 2229-4333(print), 0976-8491(online), 2011

[21].Zar Ch Su Hlaing, May Aye Lhine, “ An Ant Colony Optimization Algorithm for solving Traveling Salesman Problem", International Conference on Information Communication and management( IPCSIT), Vol,6, pp 54-59, 2011

[22].Sharvani G S and Dr. T M Rangaswamy, " Efficient Pheromone Adjustment Techniques in ACO for Ad Hoc Wireless network, IJCA(0975-8887), Vol 44-No 6, pp 29-32 April 2012.

[23].K. V. Viswanatha Cauvery N. K., "Enhanced Ant Colony Based Algorithm for Routing in Mobile AdHoc Network," World Academy of Science, Engineering and Technology, p. 46, 2008 